\title{
Wiener Polynomials for Multi-Rings Paraffin Structures
}

\author{
Ahmed M. Ali \\ ahmedgraph@uomosul.edu.iq \\ College of Computer Sciences and Mathematics \\ University of Mosul, Iraq
}

Received on: 03/09/2006

Accepted on: 24/12/2006

The distance between any two vertices $u$ and $v$ in a connected graph $\mathrm{G}$ is defined as the length of the shortest path between them, and it is denoted by $d(u, v)$. The sum of distances for all unordered pairs of distinct vertices in $\mathrm{G}$ represents Wiener index.

The number of pairs of vertices $G$ which are distance $k$ apart is denoted by $d(G, k)$, it is clear that the number of $d(G, k)$ is graphical invariant, and the Wiener polynomial of graph $\mathrm{G}$ is a generating function of the sequence $\mathrm{d}(\mathrm{G}, \mathrm{k})$.

In this paper, we find the Wiener polynomial of multi-circles of paraffin structural, and this formula which we obtained is better than the formula prove in [5], because we are able to evaluate coefficients for any limited power of $\mathrm{x}$ without depending on the number of circles, and we find the Wiener index and average distance for this structural.

Lastly, we contracted a MATLAB program to evaluate the Wiener polynomial coefficient, Wiener index and average distance.

Keywords: Wiener Polynomials , Paraffin Structures.

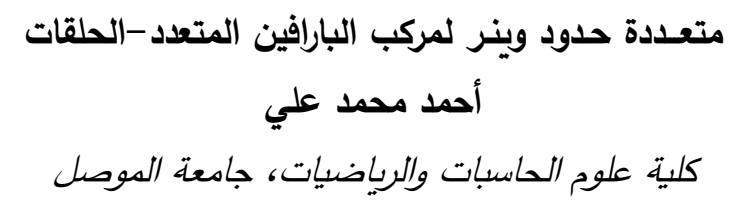

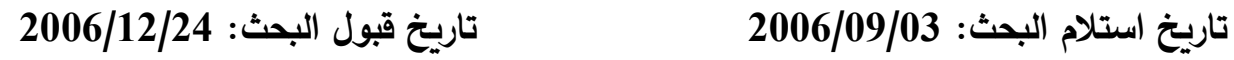

$$
\begin{aligned}
& \text { الملخص } \\
& \text { تعرف المسافة بين أي راسين v و في بيان متصل G على أنها الطول لأقصر درب بين }
\end{aligned}
$$

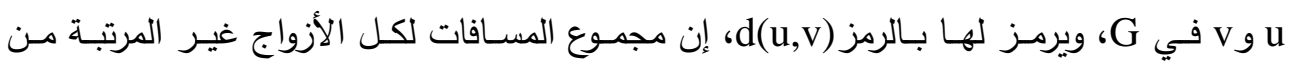

$$
\begin{aligned}
& \text { الرؤوس المختلفة في G و تمثل دليل وينر. }
\end{aligned}
$$

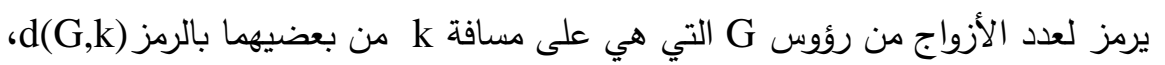

$$
\begin{aligned}
& \text { معروف أن العدد d(G,k) هو لا متغير بياني، وأن متعددة حدود وينر للبيان Gي هي علي الدالة المولدة } \\
& \text { للمتتابعة d(G,k). }
\end{aligned}
$$


في هذا البحث وضـعنا صـيغة عامـة لمتعددة حدود وينر لمركب البـارافين المتعددالحلقات. (multi-circles of paraffin structural) وهذه الصيغة التي حصلنا عليها هي أفضل من الصيغة التي وجدت في [5]، وذلك للتمكن من إيجاد المعامل لأي قوى معينة لـ x دون

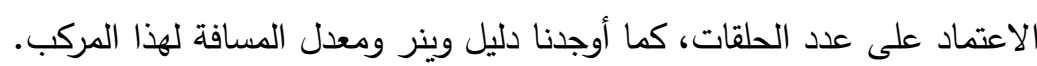
وأخيرا تم إنشاء برنامج بنظام MATLAB لاحتساب معاملات متعددة حدود وينر ودليل وينر ومعدل المسافة لهذا المركب.

الكلمات المفتاحية: متعددة حدود وينر ، مركب البارافين. المقدمة:

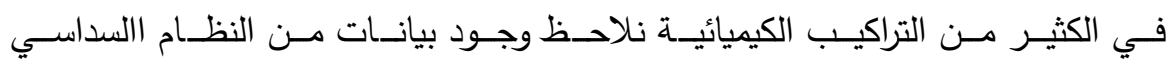

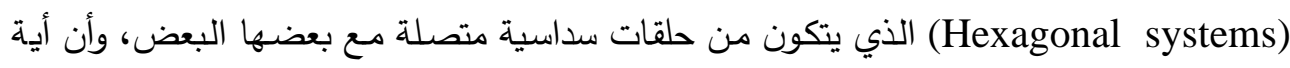
حلقتين في هذا النظام إما أن تكون مشتركة بحافة أو غير مشتركة.

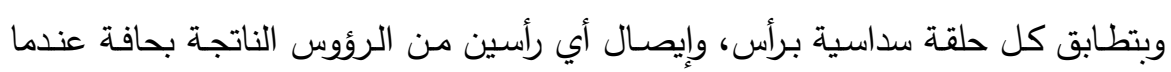

تكون الحلقتان مشتركتين بحافة، فأن البيان الناتج يسمى البيان المميز (Characteristic graph) للنظام السداسي. ويقال للنظام السداسي بـ (Catacondensed) إذا لم تكن هناك ثلاث حلقات سداسية تشترك برأس واحد. إن أنواعا من الأنظمة (Catacondensed) السداسية غير المتفرعة تحتوي على بيانات

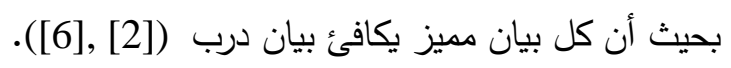
تعريف: يقال لنظـام الحلقة السداسية (Catacondensed) غير المتفرع : بأنه مركب البارافين

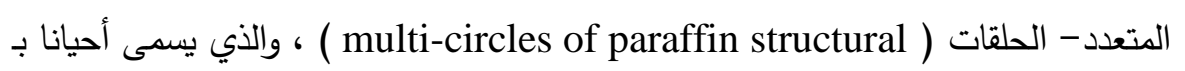
إذا كانت كل حافة مشتركة بين أية حلقتين تكون (Hexagonal ring band graph) مناظرة لكل الحافات المشتركة بين أية حلقتين النظام نفسه، كما مبين في الثكل -1-

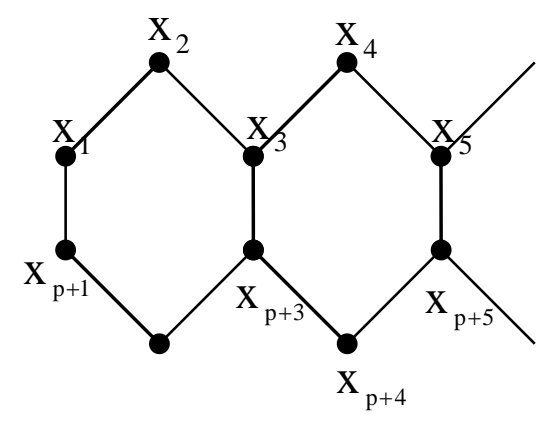

32

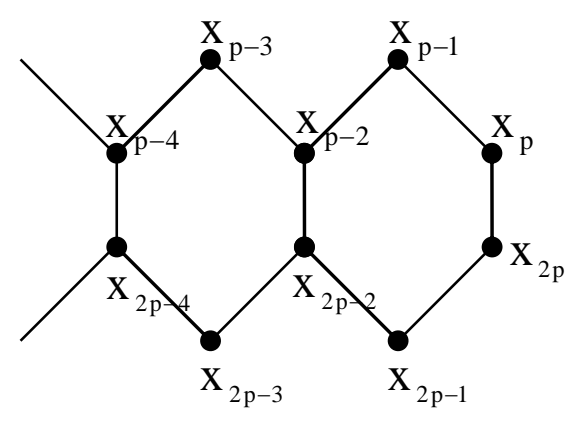




$$
X_{p+2}
$$$$
\text { الثكل -1-- }
$$

مركب البارافين المتعدد-الحلقات

وسوف نرمز لهذا النوع من المركبات بـ

والتي تقابل رتبة البيان، وأن p دائما هو عدد فردي.

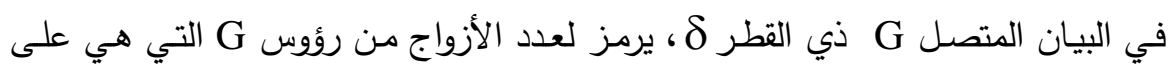

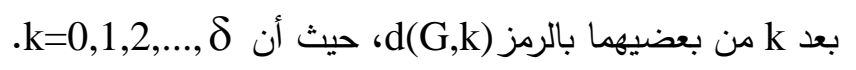

إذ تعرف متعددة حدود وينر [Wiener Polynomial)[3 للبيان G على أنها:

$W(G ; x)=\sum_{k=0}^{\delta} d(G ; k) x^{k}$

ويعرف دليل وينر (Wiener Index) للبيان G: بأنه مجموع المسافات بين كل أزواج الرؤوس في

$W(G)=\sum_{\{u, v\}} d(u, v)$

إذ أن المجموع يؤخذ على كل رأسين مختلفين u و v في G.

و يمكن التعبير عن دليل وينر بالاعتماد على متعددة حدود وينر بأنه مشتقة متعددة حدود

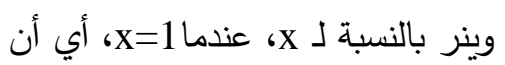

$W(G)=\left.W^{\prime}(G ; x)\right|_{x=1}=\sum_{k=1}^{\delta} k d(G ; k)$

حيث أن ${ }^{\prime}{ }^{\prime}(G ; x)$ يمثل مشتقة متعددة حدود وينر.

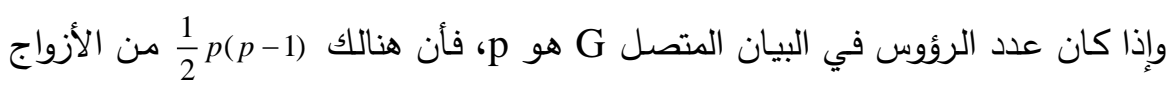
غير المرتبة للرؤوس المختلفة في البيان G. يمكننا الآن أن نعرف معدل المسافة average.

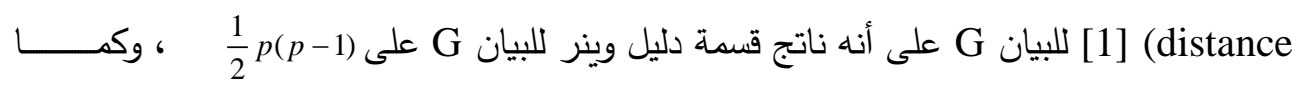
يرمز لمعدل المسافة للبيان G بـ (G)

$\bar{D}(G)=\frac{W(G)}{p(p-1) / 2}$ 
ولا بـد لنا أن نذكر أن العـالم [3.Wiener كان أول من درس المسافة الكلية في

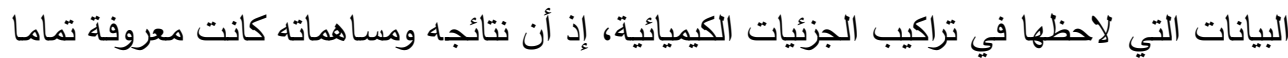
لعلماء الكيمياء المختصين ولكن معرفة علماء الرياضيات بها كانت محدودة. وأن الفائدة من متعددة حدود وينر هي للحصول على المسافة الكلية والتي تمثل دليل وينر (Wiener index)، والذي لهاء لهاه استخدامات واسعة في الكيمياء [1].

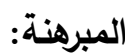

إن متعددة حدود وينر لمركب البارافين المتعدد- الحلقات

$$
W\left(H_{2 p} ; x\right)=\sum_{k=0}^{\delta} d\left(H_{2 p}, k\right) x^{k} ;
$$

$d\left(H_{2 p}, 0\right)=2 p$

حيث أن

$d\left(H_{2 p}, 1\right)=\frac{1}{2}(5 p-3)$;

$d\left(H_{2 p}, k\right)=4(p-k)+2+R ; \quad k=2,3, \ldots, \delta$

$$
\mathrm{R}=\left[\begin{array}{cc}
(\mathrm{p}-1) / 2 & \text { when } \mathrm{k}=3 \\
0 & \text { other wise }
\end{array}\right.
$$

حيث أن

$V_{1}=\left\{x_{1}, x_{2}, \ldots, x_{p}\right\}$

$V_{2}=\left\{x_{p+1}, x_{p+2}, \ldots, x_{2 p}\right\}$

سوف نرمز للمسافة بين أي رأسين في

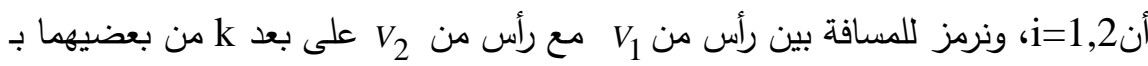
$\cdot d\left(H_{2 p}, k\right) V_{1} V_{2}$

$d\left(H_{2 p}, k\right)_{V_{i}}=d\left(P_{p}, k\right)=p-k \quad ; \quad i=1,2$

نلاحظ أن 
من الواضح أن

$$
\begin{aligned}
d\left(H_{2 p}, 1\right) & =d\left(H_{2 p}, 1\right)_{1}+d\left(H_{2 p}, 1\right) V_{2}+d\left(H_{2 p}, 1\right) V_{1} V_{2} \\
& =(p-1)+(p-1)+\frac{1}{2}(p+1)=\frac{1}{2}(5 p-3)
\end{aligned}
$$

$d\left(H_{2 p}, 1\right) V_{1} V_{2}=\sum_{i=1}^{(p+1) / 2} d\left(x_{2 i-1}, H_{2 p}, 1\right) V_{2}=\sum_{i=1}^{(p+1) / 2}=\frac{1}{2}(p+1) \quad$ حيث أن

الآن نتبع طريقة الاستقراء الرياضي لإثبات العلاقة (1).

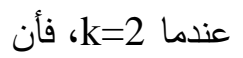

$$
\begin{aligned}
d\left(H_{2 p}, 2\right) & =d\left(H_{2 p}, 2\right) V_{1}+d\left(H_{2 p}, 2\right) V_{2}+d\left(H_{2 p}, 2\right) V_{1} V_{2} \\
& =(p-2)+(p-2)+2+2(p-2)=4(p-2)+2
\end{aligned}
$$

حيث أن

$$
\begin{aligned}
d\left(H_{2 p}, 2\right) V_{1} V_{2} & =\sum_{i=1}^{p} d\left(x_{i}, H_{2 p}, 2\right) V_{2}=1+\sum_{i=2}^{p-1} d\left(x_{i}, H_{2 p}, 2\right) V_{2}+1 \\
= & 1+\sum_{i=2}^{p-1} 2+1=2+2(p-2)
\end{aligned}
$$

عندما k=3أن

$$
\begin{aligned}
d\left(H_{2 p}, 3\right) & =d\left(H_{2 p}, 3\right) V_{1}+d\left(H_{2 p}, 3\right) V_{2}+d\left(H_{2 p}, 3\right) V_{1} V_{2} \\
& =(p-3)+(p-3)+4+2\left(\frac{p-1}{2}\right)+3\left(\frac{p-5}{2}\right) \\
& =4(p-3)+2+(p-1) / 2
\end{aligned}
$$

$$
\begin{aligned}
d\left(H_{2 p}, 3\right) V_{1} V_{2} & =\sum_{i=1}^{p} d\left(x_{i}, H_{2 p}, 3\right) V_{2}=1+2+\sum_{i=3}^{p-2} d\left(x_{i}, H_{2 p}, 3\right) V_{2}+2+1 \\
& =1+2+\sum_{j=2}^{(p-1) / 2} d\left(x_{2 j-1}, H_{2 p}, 3\right) V_{2}+\sum_{j=2}^{(p-3) / 2} d\left(x_{2 j}, H_{2 p}, 3\right) V_{2}+2+1 \\
& =3+\sum_{j=2}^{(p-1) / 2(p-3) / 2} \sum_{j=2}^{\sum 3+3}=4+2\left(\frac{p-1}{2}\right)+3\left(\frac{p-5}{2}\right)
\end{aligned}
$$

عندما k=4، فأن

$$
\begin{aligned}
d\left(H_{2 p}, 4\right) & =d\left(H_{2 p}, 4\right)_{V_{1}}+d\left(H_{2 p}, 4\right)_{V_{2}}+d\left(H_{2 p}, 4\right)_{V_{1}} V_{2} \\
& =(p-4)+(p-4)+2+2(p-4)=4(p-4)+2
\end{aligned}
$$




$$
\begin{aligned}
& \text { حيث أن } \\
& d\left(H_{2 p}, 4\right) V_{1} V_{2}=\sum_{i=1}^{3} d\left(x_{i}, H_{2 p}, 4\right) V_{2}+\sum_{i=4}^{p-3} d\left(x_{i}, H_{2 p}, 3\right) V_{2}+\sum_{i=p-2}^{p} d\left(x_{i}, H_{2 p}, 3\right) V_{2} \\
& =\sum_{i=1}^{3} 1+\sum_{i=4}^{p-3} 2+\sum_{i=p-2}^{p} 1=3+2(p-6)+3=2+2(p-4) \\
& \text { نغرض أن الصيغة (1) صحيحة عندما k=r) أي أن } \\
& d\left(H_{2 p}, r\right)=4(p-r)+2 \\
& \text { ألان سوف نبرهن أن الصيغة (1) صحيحة عندما } \\
& d\left(H_{2 p}, r+1\right)=d\left(H_{2 p}, r+1\right) V_{1}+d\left(H_{2 p}, r+1\right) V_{2}+d\left(H_{2 p}, r+1\right) V_{1} V_{2} \\
& =(p-(r+1))+(p-(r+1))+2+2(p-(r+1)) \\
& =4(p-(r+1))+2 \\
& \text { حيث أن } \\
& d\left(H_{2 p}, r+1\right) V_{1} V_{2}=\sum_{i=1}^{r} d\left(x_{i}, H_{2 p}, r+1\right) V_{2}+\sum_{i=r+1}^{p-r} d\left(x_{i}, H_{2 p}, r+1\right) V_{2} \\
& +\sum_{i=p-r+1}^{p} d\left(x_{i}, H_{2 p}, r+1\right) V_{2} \\
& =\sum_{i=1}^{r} 1+\sum_{i=r+1 i=p-r+1}^{p-r} 2+\sum_{i=1}^{p} 1=r+2(p-2 r)+r \\
& =2(p-(r+1))+2
\end{aligned}
$$

النتيجة:

دليل وينر لمركب البارافين المتعدد- الحلقات

$W\left(H_{2 p}\right)=\frac{1}{3} p(p+1)(2 p+1)-1$.

وأن معدل المسافة لهذا المركب لا يزيد على (3+3) •

البرهان:

$$
\begin{aligned}
W\left(H_{2 p}\right)=\left.W^{\prime}\left(H_{2 p}, x\right)\right|_{x=1} & =\frac{1}{2}(5 p-3)+8(p-2)+4+12(p-3)+6 \\
& +\frac{3}{2}(p-1)+\sum_{k=4}^{p} 4 k(p-k)+2 k
\end{aligned}
$$

ويمكن كتابة الصيغة أعلاه بالثكل الاتي 


$$
\begin{aligned}
W\left(H_{2 p}\right) & =-1+\sum_{k=1}^{p} 4 k(p-k)+2 k \\
& =-1+2(2 p+1) \sum_{k=1}^{p} k-4 \sum_{k=1}^{p} k^{2} \\
& =-1+2(2 p+1)\left(\frac{p(p+1)}{2}\right)-4\left(\frac{p(p+1)(2 p+1)}{6}\right) \\
& =-1+\frac{1}{6} p(p+1)(2 p+1)(6-4)=\frac{1}{3} p(p+1)(2 p+1)-1
\end{aligned}
$$

$\bar{D}\left(H_{2 p}\right)=\frac{W\left(H_{2 p}\right)}{2 p(2 p-1) / 2}$

$$
=\frac{p(p+1)(2 p+1)-3}{3 p(2 p-1)}
$$

وأن معدل المسافة

$\bar{D}\left(H_{2 p}\right)=\frac{1}{3}(p+2)+\frac{(p-1)}{p(2 p-1)}$

نلاحظ أن المقدار

$$
\bar{D}\left(H_{2 p}\right)<\frac{1}{3}(p+2)+\frac{1}{3}=\frac{1}{3}(p+3)
$$

أكبر أو يساوي 3 في هذه المركبات).

وبعد تبسيط المقدار نحصل على

حيث أن المقدار (3+3)

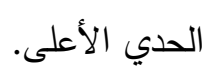

فإذا أخذنا قيمة p مثلا هي 7 فأن

$W\left(H_{14} ; x\right)=14+16 x+22 x^{2}+21 x^{3}+14 x^{4}+10 x^{5}+6 x^{6}+2 x^{7}$

$W\left(H_{14}\right)=279$

$\bar{D}\left(H_{2 p}\right)=279 / 91 \cong 3.0659<3.3333$

وأخيـرا صـممنا برنـامج بلغـة MATLAB لاحتسـاب المعـاملات لمتعـدة حـدود وينـر

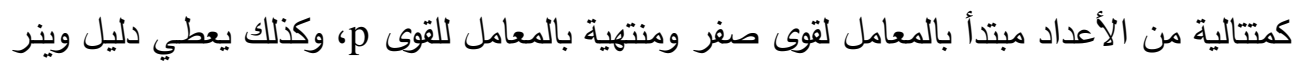

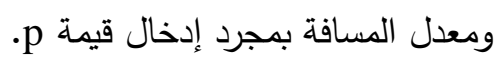

وللتأكد من دقة البرنامج أدخلنا p=7 فكانت النتائج مطابقة، ابتداء من المعاملات لمتعددة

حدود وينر ، دليل وينر ومعدل المسافة. 


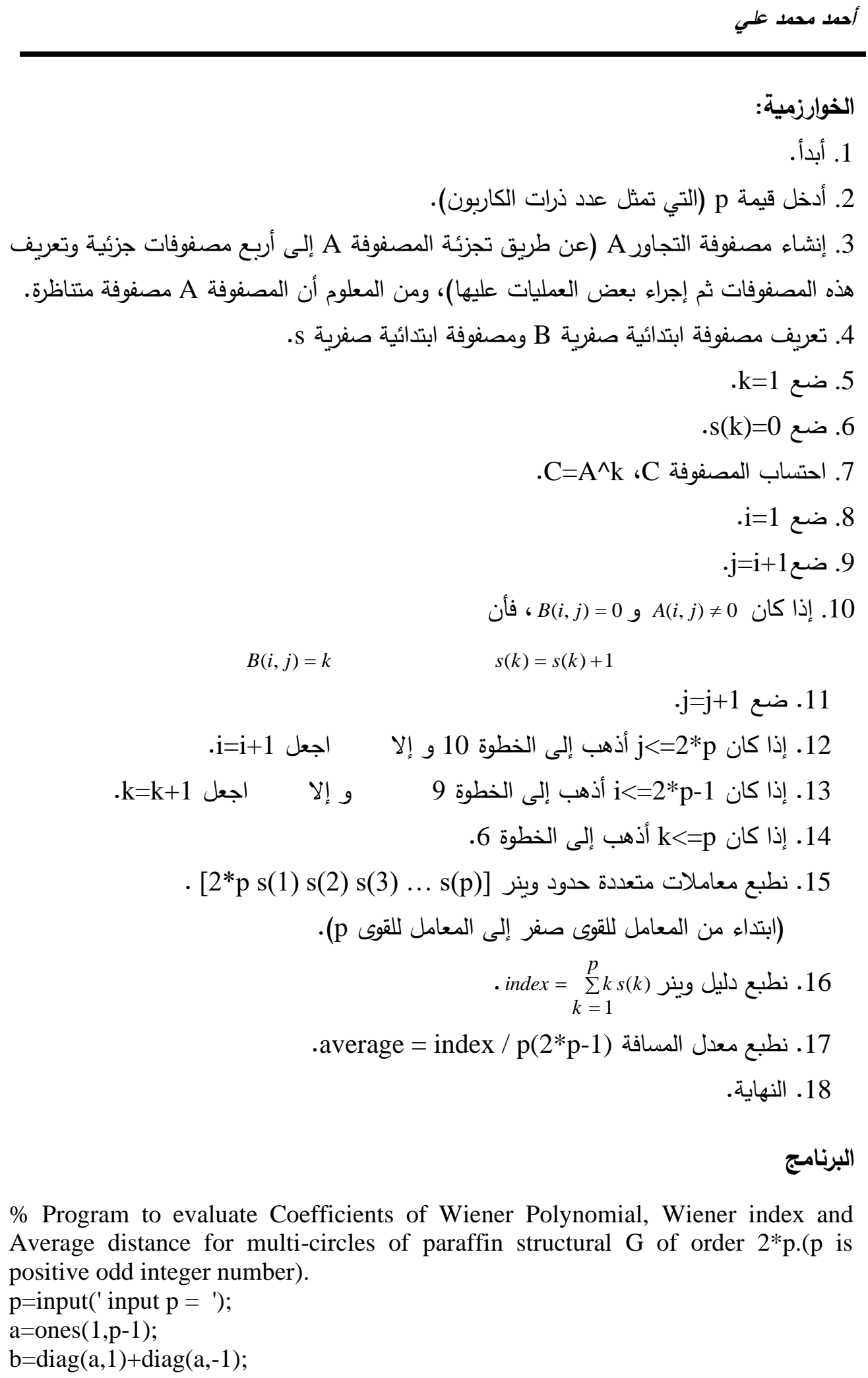

$\%$ Program to evaluate Coefficients of Wiener Polynomial, Wiener index and Average distance for multi-circles of paraffin structural $G$ of order $2 * p .(p$ is positive odd integer number).

$\mathrm{p}=$ input(' input $\mathrm{p}=$ ');

$\mathrm{a}=$ ones $(1, \mathrm{p}-1)$;

$\mathrm{b}=\operatorname{diag}(\mathrm{a}, 1)+\operatorname{diag}(\mathrm{a},-1)$; 


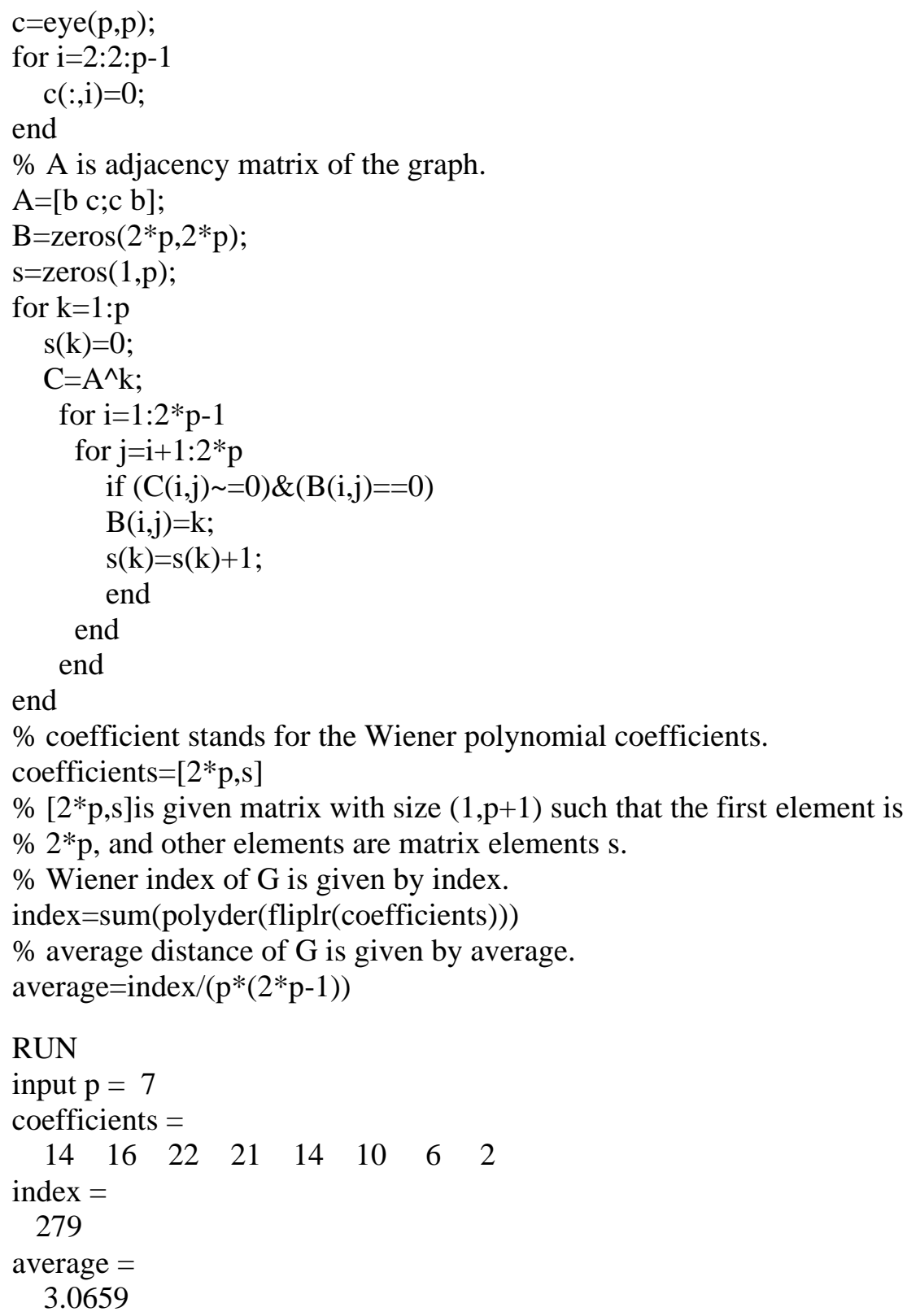




\section{المصادر}

[1] J.I. Doyle and J.E. Graver (1977) "Mean distance in a graph“, Discrete Math., Vol. 17, PP.147-154.

[2] A.A. Dobrynin (1993) "On decomposition of the Wiener index for Graphs of Catacondensed Hexagonal Systems", Graph Theory Notes of New York; XXV. PP.19-21.

[3] I. Gutman (1993) "Some properties of the Wiener polynomial", Graph Theory Notes of New York; XXV. PP.13-18.

[4] H. Hosoya (1988) "On some counting polynomials in chemistry", Discrete Applied Math. 19,PP.239-257.

[5] Walid A.M. Saeed (1999) Wiener Polynomials of Graphs, Ph.D. Thesis , Mousl University.

[6] H. Wiener (1947) "Structural determination of paraffin boiling points", J. Amer. Chem. Soc., 69 , PP.17-20. 